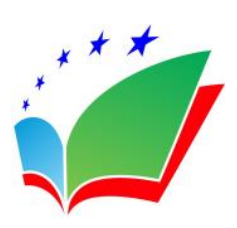

European Journal of Fitness, Nutrition and Sport Medicine Studies

ISSN: 2668 - 9758

ISSN-L: 2668 - 9758

Available on-line at: $\underline{\text { www.oapub.org/hlt }}$

\title{
AGING \& MUSCULAR FUNCTION: \\ A SELECTED REVIEW OF LITERATURE WITH EMPHASIS ON CARDIORESPIRATORY ENDURANCE AND FUNCTIONAL PERFORMANCE RESPONSE TO RESISTANCE EXERCISE
}

\author{
Ozan Atalağ \\ Cem Kurt ${ }^{2 i}$, \\ Lincoln A. Gotshalk ${ }^{1}$, \\ Richard E. J. Shanklin', \\ Jenna H. Aina ${ }^{1}$, \\ Ian McQuate ${ }^{1}$ \\ ${ }^{1}$ Department of Kinesiology and Exercise Sciences, \\ University of Hawai'i at Hilo, \\ Hilo, HI, USA \\ ${ }^{2}$ Department of Coaching Education, \\ School of Kirkpinar Physical Education and Sports, \\ Trakya University, \\ Edirne, Turkey
}

\begin{abstract}
:
This narrative review evaluates strength or resistance training on cardiorespiratory endurance, blood pressure, contractile function, contractile protein synthesis rate, bone turnover, gait and balance, and neuromuscular adaptations in elderly populations. Seventy-eight studies spanning from 1999 through 2020 were reviewed. Database sources including PubMed, Science Direct, Web of Knowledge and Google Scholar were searched in accordance with the purpose of the study. A majority of the studies reported that resistance training reduces blood pressure and increases contractile functions, contractile protein synthesis rate, bone turnover, gait and balance, cardiorespiratory endurance, and neuromuscular adaptations in the elderly. Furthermore, combined training (CT), also known as concurrent training (strength plus endurance training) may also be as effective as traditional endurance training or traditional strength/resistance training alone for improving cardiorespiratory endurance and functional performance. According to the evaluation of studies included in this review, we concluded that training modalities that involve low-load, high velocity strength training combined with endurance training might be the best training strategy in improving cardiovascular fitness, functional capacity and musculoskeletal health in the elderly populations. Elderly people should be
\end{abstract}

i Correspondence: email cemkurt35@gmail.com 
encouraged to participate in a concurrent training or a combination of strength and endurance training to delay, or even reverse the negative effects of aging.

Keywords: balance, born turnover, cardiovascular endurance, elderly, gait, neuromuscular adaptations

\section{Introduction}

\subsection{What is biological aging?}

Aging is a complex multifactorial biological process shared by all living organisms. It is manifested by a gradual decline of normal physiological functions in a time-dependent manner (Pal and Taylor,2016, pp.1). The underlying physical conditions associated with biological aging include an increased risk of higher blood pressure, loss of muscle mass, loss of strength and contractile function, a decrease in bone turnover rates, gait speed, balance, cardiovascular endurance, and neuromuscular adaptability (Ferruci et al., 2014; Jaul \& Barron, 2017; Navaratnarajah \& Jackson, 2017). These patterns of aging eventually result in a loss of independence and the inability to perform daily activities (Allen et al., 2013; Gschwind et al., 2013). Furthermore, studies also indicate that age-related muscle loss (sarcopenia) and loss of strength can cause functional decay (Ferruci et al., 2017; Finger at al., 2015; Landi et al., 2017; Wood et al., 2001).

The physical states during aging, or biological aging, have traditionally been linked to the number of years of life an organism has aged, also known as chronological aging (Hamczyk et al., 2020) Within the aging population, distinctions are made based on age groups such as "young old" (65-74 years old), "middle old" (75-85 years old) and the "oldest old" (85 years and older). However, human aging is generally considered to begin after a period of near total cellular renewal lasting 20-35 years (Blumental et al., 2013), and aging research begins thereafter.

\subsection{Objectives}

As more factors that are associated with the effects of aging are being discovered, it has begun to be viewed as a disease, which is caused by disuse, or genetic factors, and thus considered potentially treatable. Research on aging is of great importance due to the presence of an extremely large aging population. It is predicted that the population of adults 65 and older in America, will increase from 35 million to 72 million by 2030 (Allen et al., 2013). Due to the increase of an aging generation known as the "baby boomers," much of the research has been focused on possible ways that will delay, mitigate, or even reverse negative effects of aging (Distefano \& Goodpaster, 2018; Rebelo-marques et al., 2018; Ren \& Zhang, 2018; Ros \& Carrascosa, 2020)

Along with growing interest in the mechanisms of aging, the effects of resistance training have been investigated extensively as well (Fragala et al., 2019; Guizelini et al., 2018; Kraschnewski et al., 2016; Lacroix et al., 2016;). Traditionally, resistance training has 
been associated with select populations such as athletes, body builders and the younger population (Kraemer et al., 2004). Resistance training participation as a form of therapeutic treatment in elderly population is roughly around $11 \%$ of the population (Centers for Disease Control and Prevention, 2004). Despite of this relatively low participation, The American College of Sports Medicine and American Heart Association recommend that resistance training be performed due to the benefits it can provide for the elderly population (Kraemer et al., 2004).

This paper is a brief presentation of literature regarding research that examines the relationship between the effects of resistance training and the process of aging. The inclusion criteria for the studies in this paper involved the presence of primary information pertaining to the human anatomical structures and physiological processes that are considered to be causative agents in the process of aging. Secondly, the selected studies must have implemented a resistance exercise program that had the potential to defer, retard, reverse, or in some manner provide a positive improvement upon the declines that accompany aging (Allen et al., 2013; Gschwind et al., 2013).

\section{Selected Literature}

The number of studies in human aging is prolific and continues to grow due to the many variables and factors that are associated with aging. The rapid growth of an aging population around the globe is presenting a need to understand the future implications of this phenomenon and the ways it will have an effect on a multitude of areas such as health care system, the economy, and politics to name a few. The specific studies presented were selected from among a vast and growing body of literature, and thus cannot be considered comprehensive. The reviewed literature included was chosen, instead, to convey an idea of the scope of negative effects of aging in humans which can be influenced by resistance training. Exact sections of the original article(s) or abstract(s) are presented in order to preserve the integrity of the literature as much as possible. Database search was based on the University of York's Centre of Reviews and Dissemination without any limitation on the publication date. The databases used for this review included PubMed, Science Direct, Web of Knowledge and Google Scholar. Although relatively recent studies (last 5 years) were preferred to make a citation, where required, older studies were cited as well. The following search keywords were used: "strength training AND elderly" and "resistance training AND elderly", "training AND elderly", "sarcopenia", "bone turn over AND elderly", "muscle force AND elderly", "fall prevention AND elderly", "Strength training AND prevention", "cardiorespiratory endurance AND elderly", "strength training AND functional capacity". 144 studies, spanning from 1999 to 2020 were retrieved of which 78 met the inclusion criteria.

Database search procedure is shown in Figure I. The inclusion criteria for study selection were: (1) the literature was written in English, (2) subjects were elderly who were either healthy or displayed sarcopenia, osteoporosis, heart disease or obesity, and (3) 
female and male participants who were $\geq 65$ years old. The exclusion criteria included: 1 ) No access to full text 2) Language of publication is not English 3) A medical intervention is used with resistance training

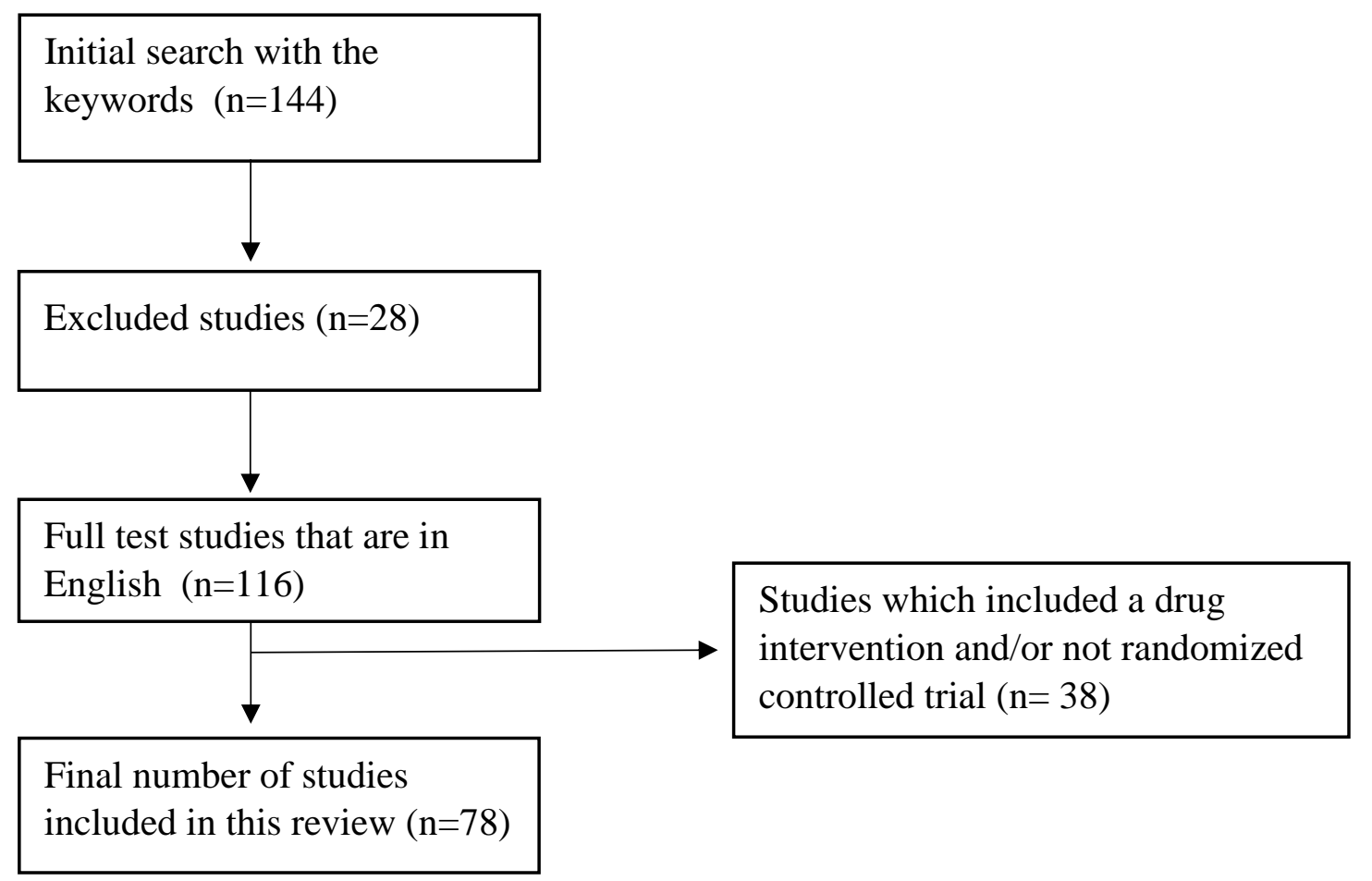

Figure 1: Flow diagram of narrative review of literature

\subsection{Cardiorespiratory Endurance}

Cardiorespiratory endurance refers to the ability of the heart and lungs to deliver oxygen to working muscles during continuous physical activity and it is an important indicator of physical health (Cheng, Chiu, \& Su, 2019). Pollock et al. (1998) recommended physical activities that involve large muscle groups (walking, jogging, swimming, cycling, rope skipping etc.), done at $50 \%$ - $90 \%$ of maximum heart rate intensity, 3-5 days/week, either continuously or intermittently for 20-60 min, for improving cardiorespiratory endurance. Traditionally, the majority of training studies have used aerobic modalities to improve cardiorespiratory endurance and have not specifically targeted skeletal muscle or used resistance training (Cheng, Chiu \& Su, 2019; Maiorana et al., 2001; Pollock et al., 1998). Furthermore, resistance training has not been studied as often, especially in patients with chronic heart failure, due to the assumption of producing cardiac overload, hemodynamic burden, decreased myocardial perfusion, or causing wall motion abnormalities or arrhythmias (Maiorana et al., 2001). However, the American Heart Association and the American College of Sport Medicine state that resistance training is an effective method to increase muscle mass, and may reduce cardiovascular disease (Braith, 2006).

Accordingly, a study done by Vincent et al. (2002) showed that high intensity resistance training is as effective as low intensity resistance training to improve cardiovascular endurance. The study compared the effects of 6 months of high or low- 
intensity resistance exercise on aerobic capacity and treadmill time to exhaustion in 62 adults aged 60 to 83 years old. Authors (2002) reported that 1-RM for all exercises, time to exhaustion at treadmill test and aerobic capacity increased significantly $(p \leq .05)$ for both groups showing that even high intensity resistance training might increase cardiovascular capacity.

Similarly, in a study done by Frontera et al. (1990), strength training done for 12 weeks increased maximal aerobic capacity $\left(\mathrm{VO}_{2} \max \right)$ and some of its determinants such as hematological markers, and muscle oxidative capacity in healthy older men (60-72 years). Authors reported that increase in aerobic capacity in subjects who underwent resistance training might have been caused by oxidative enzyme activities (citrate synthase) and increase in capillaries per fiber.

Combined training (CT), also known as the concurrent training (strength plus endurance training) is a relatively new exercise modality for improving cardiorespiratory endurance in elderly. In a recent systematic review and meta-analysis which was published by Hurst et al. (2019), it was reported that CT may be more effective than traditional endurance training or resistance exercise alone for cardiorespiratory fitness improvement. Their results were also in line with the study done by Cadore et al. (2018), showing that CT improved maximal and submaximal endurance performance in healthy elderly men (64.7 \pm 4.1 years) who performed different variations of $\mathrm{CT}$, three times a week for 12 weeks.

However, some studies also showed that concurrent strength and endurance training typically lead to similar improvements in maximal aerobic performance (i.e., $\mathrm{VO}_{2 \text { peak }}$ ) compared with endurance training alone in elderly populations (Cadore et al., 2010; Holviala et al., 2010; Karavirta et al., 2011; Izquierdo et al., 2004; Wood et al., 2001)

To our understanding, as shown in studies presented above, CT may be more effective than traditional endurance training or resistance exercise alone for cardiorespiratory endurance improvement in elderly.

\subsection{Blood Pressure}

More than half of the 55-year-old population is expected to develop hypertension within 10 years (Olher et al., 2013). Furthermore, high blood pressure is one of the nine leading factors that contribute to cardiovascular disease, which causes more than 7 million+ deaths each year (Cornelissen et al., 2001).

Fagard (2011) suggests that hypertensive patients should be advised to participate in at least 30 min of moderate-intensity dynamic aerobic exercise (walking, jogging, cycling or swimming) for 5-7 days per week. When it comes to resistance training, dynamic RT exercises recommended by The American College of Sports Medicine and European Society of Hypertension / European Society of Cardiology are, moderate intensity (60-80 \% of 1 RM), 8-12 repetitions (Pescatello et al., 2015) and should be done 23 days/week. Although some authors argued that isometric RT exercises can be used for reducing resting blood pressure (Wiles et al., 2018; Inder et al., 2016), they are not 
recommended since data is only available from a small number of studies and is limited (Mancia et al., 2013 Pescatello et al., 2015;Williams et al., 2018; ).

Da Cunha Nascimento et al., (2014) conducted a study to determine the effects of moderate intensity resistance training on resting blood pressure of 20 sedentary hypertensive elderly (67.6 \pm 6.4 years). In the study subjects performed two times per week over 14 weeks, consisting of three sets with a training zone of eight to twelve repetitions, at a moderate intensity, using a modified version of perceived exertion (1-10 point scale), and their resting blood pressure was measured before and after exercise. The resistance training program led to decrease of systolic (pre training 130.60土 $8.05 \mathrm{mmHg}$ versus post training $112.50 \pm 9.66 \mathrm{mmHg}, \mathrm{p}=0.001$ ), diastolic (pre training $80.60 \pm 7.75 \mathrm{mmHg}$ versus post training $70.50 \pm 9.51 \mathrm{mmHg}, \mathrm{p}=0.008$ ), and mean blood pressure (pre training 93.70 \pm $6.22 \mathrm{mmHg}$ versus post training $84.50 \pm 9.11 \mathrm{mmHg}, \mathrm{p}=0.002)$ when compared to preexercise values

Other studies have reported conclusive results in longer periods of strength training using lower to moderate intensities (Braith, 2006; Cornelissen et al., 2011; da Cunha Nascimento et al., 2014; Martel et al., 1999).

A similar study by Kim \& Kim (2013) investigated the effects of long-term resistance exercise on body composition, blood lipid factors and vascular compliance in hypertensive men. A total of 35 male subjects between the ages of 68-72 were studied. All participants performed exercises on weight training machines for 30 minutes a day, for one year. body composition was measured by using bioelectrical impedance (InBody $\left.{ }^{\circledR}\right)$. Also measured were blood lipid profile and vascular compliance and blood pressure was taken pre- and post-intervention. The intensity of the resistance exercise program was at $60 \%$ of maximum voluntary contraction test with 10 repetitions. The study found that there was an improvement in lean body mass and vascular compliance, and a suppression of low density lipid-cholesterol (LDL) levels and a decrease in the percentage of body fat in the subjects, ultimately reducing the hypertension levels in these men. In conclusion, Kim \& Kim (2013) suggested that resistance exercise could be a valuable tool for treatment of hypertension.

Although resistance training has been shown to have the potential to reduce blood pressure, studies done by Braith et al. (2006) and Cornelissen et al. (2011) and concluded that a combination of endurance training and resistance training at a moderate intensity ensure the best results. When the study of da Cunha Nascimento et al. (2014) is considered, it appears that a moderate intensity seems to produce consistent results throughout. As a whole for strength training alone, reproducible evidence is limited in older populations (Cornelissen et al., 2011; Nascimento et al., 2014). Ultimately, factors that skew results include; duration of the exercise program and types of exercises that may or may not improve blood pressure.

A pilot study done by da Cunha Nascimento et al. (2014) analyzed the effects of moderate intensity resistance training for 14 weeks on hand-grip strength along with blood pressure in 12 elderly hypertensive women. Exercises emphasized ankle, hip, and 
lumbar joints. Results showed that systolic and diastolic blood pressure decreased significantly during the training period. However, there was no difference in the detraining period for diastolic blood pressure (remained 13\% lower). But the results were clinically significant for systolic and diastolic blood pressure with the effect sizes above 0.5. In the study systolic blood pressure found for pre-training and post-training as 130.60 $\pm 8.05 \mathrm{mmHg}$ and $112.50 \pm 9.66 \mathrm{mmHg}$, respectively. In the study also diastolic blood pressure determined as $80.60 \pm 7.55 \mathrm{mmHg}$ and $70.50 \pm 9.51 \mathrm{mmHg}$ for pre-training and post-training, respectively.

Dantas et al. (2016) reported that a dynamic strength training program taking place 2-3 times per week over 10 weeks led to a reduction in the oxidative stress of the in hypertensive elderly women (mean age $=66.1$ years). Additional cardiovascular benefits included an increase in forearm vasodilatation and a reduction in mean blood pressure, both at rest and during static handgrip exercise.

According to the above presented literature, dynamic strength training is effective in reducing or controlling blood pressure in the elderly. However, the effects of isometric resistance training on blood pressure remain unclear.

\subsection{Contractile Function}

Decreases in skeletal muscle strength and size are characteristics commonly associated with old age and it has been shown that the contractile velocity of a single muscle cell can decrease by as much as $40 \%$ in older populations when compared to younger individuals. In addition, age related muscle mass loss is associated with detrimental outcomes in the elderly population (Taekema et al., 2010). Furthermore, a lower peak $\mathrm{VO}_{2}$ max along with decrease of tissue blood supply and $\mathrm{O}_{2}$ extraction lower the threshold at which anaerobic metabolism dominates energy production, which consequently leads to early onset of fatigue (Allen et al., 2013)

A study conducted by Trappe et al. (2000), examined whether the contractile function at the single fiber level is altered with resistance training in older adults. The study involved seven older men (age: age $=74 \pm 2 \mathrm{yr}$ ) who performed progressive resistance training (PRT) with the knee extensor muscle group. They performed at $80 \%$ of 1RM, 3 days/week for 12 weeks. Trappe et al. (2000) concluded that PRT in elderly men increased muscle cell size, strength, contractile velocity, and power in both slow- and fasttwitch muscle fibers.

The effects of high velocity resistance training (HVR) for improving muscle power when compared to traditional low velocity training (LVR) are not clear (Grey et al., 2018; Hazell, Kennoo \& Jakobi, 2007; Reid \&Fielding, 2012; Trappe et al., 2000). Some authors argued that peak muscle power is typically yielded at approximately $70 \%$ of the $1 \mathrm{RM}$ while maximal contraction velocity typically occurs at the lowest external resistance (40\% of 1RM) (Callahan et al., 2007; Cuoco et al., 2004; Fielding et al., 2002).

Hazell, Kenno \& Jakobi (2007) reported that older adults should perform Resistance Training (RT) with heavier loads (60\% of 1-RM) to increase muscle power. 
Reid et al. (2012) conducted an observational cohort study in older adults ( $\geq 65$ years), and they concluded that peak muscle power has emerged as an important predictor than muscle strength for functional limitations in older adults. Reid \& Fielding (2012) conducted randomized trials of skeletal power as a critical determinant of physical functioning in older adults which demonstrated that high velocity resistance training is more effective for improving muscle power compared to traditional slow velocity training. In general, high velocity resistance training for power improvements is safe and well tolerated even in older adults with limited mobility and among populations over 80 years. On the other hand, Grey et al. (2018) reported that high velocity resistance training with $50 \%$ of $1 \mathrm{RM})$ and low velocity resistance training ( $80 \%$ of $1 \mathrm{RM})$ have similar effects in improving body composition in a 48 week program. Although HV participants increased total strength by $27 \%$ in the first 24 weeks, results of this study revealed that LV is more effective in increasing muscle strength than HVL 24 to 48 weeks ( $n=99$ female and male, age $=81.2 \pm 5.6$ years). The same study also showed that high velocity resistance training is more effective for improving physical function that was determined via the senior fitness test battery (SFT) which included upper- and lower-body strength, agility, cardiovascular endurance and upper- and lower-body flexibility.

The results of a systematic meta-analysis suggest that there is inconclusive evidence to support the superiority of fast-intended-velocity resistance training to improve functional capacity when compared to moderate-velocity or slow velocity resistance training with participants above 60 years old (da Rosa Orssatto et al., 2019). On the other hand, a recent position statement by the National Strength and Conditioning Association posits that resistance training should include power exercises performed at high velocities in concentric movements with moderate intensities (i.e., $40-60 \%$ of $1 \mathrm{RM}$ ) to benefit functional improvements (Fragala et al., 2019). Compared to LVR, it has been suggested that HVR leads to higher neuromuscular performance defined as increased high-velocity tension and rate coding (motor-unit firing frequency), increased inhibition of antagonist muscles, a better co-contraction of synergistic muscle, an increased activation of synergistic muscles, an increased motor neuron excitability (Bottaro et al., 2007; Komi, 1984; Lyttle, Wilson \& Ostrowski, 1996;).

\subsection{Contractile Protein Synthesis Rate}

Aging is associated with a loss of skeletal muscle mass at a rate of $0.5-2 \%$ per year (Kumar et al., 2009). Sarcopenia is defined as a loss of muscle strength/power with ageing due to changes in the quality and quantity of muscle tissue (Reid \& Fielding, 2012). In the absence of a disease state or injury, sarcopenia results in a $3-8 \%$ reduction in muscle mass per decade (Paddon-Jones et al., 2015). Two prophylactics suggested to curtail the loss of muscle mass are resistance exercise and dietary-protein. Resistance exercise is a potent stimulus to increase muscle strength, physical functioning and to preserve muscle mass because of the mechanic loading component (Stec et al., 2017) and ingestion of protein acts as a major stimulus for muscle protein synthesis (Bowden Davies et al., 2019). 
A study by Hasten et al. (2000) examined the short-term (2 weeks) effects of weight training on the synthesis rate of the major contractile proteins (myosin heavy chain or MHC, actin, and mixed muscle proteins) in paired young and elderly subjects (including both women and men) who were considered healthy. Fractional synthesis rates of mixed, $\mathrm{MHC}$, and actin proteins were determined in seven healthy, sedentary 23 to 32-year-old and seven healthy 78 to 84 year old participants before and after a two-week weight training program. The in vivo rate of incorporation of $1-[(13 C)]$ leucine into vastus lateralis $\mathrm{MHC}$, actin, and mixed muscle proteins were determined. Before starting the intervention, mixed and MHC fractional synthesis rates were lower in the older group than they were in younger participants $(p<0.04)$. Baseline actin protein synthesis rates were similar in the two group $(\mathrm{p}>0.05)$. Over a two-week period, participants completed ten 1- to 1.5-hour resistance training sessions that consisted of 2-3 sets per day of 9 exercises, with 8-12 repetitions per set, at $60-90 \%$ of maximum velocity muscle strength. At the end of the 2 week period, MHC and mixed protein synthesis rates increased both in the younger (88\% and $121 \%)$ and the older participants $(105 \%$ and $182 \%$; $<0.001$ vs. baseline). These findings indicate that MHC and mixed protein synthesis rates are reduced more than actin with advanced age. Similar to that of the 23-32-year-old subjects, the vastus lateralis muscle in the 78-84-year-old subjects appears to retain the capacity to increase $\mathrm{MHC}$ and mixed protein synthesis rates in response to short-term resistance exercise (Hasten et al., 2000).

On the another hand, Villareal et al. (2011) aimed to determine the effects of a 3month long multicomponent exercise training program consisted of $15 \mathrm{~min}$ of flexibility exercises, followed by $30 \mathrm{~min}$ of endurance exercise, $30 \mathrm{~min}$ of strength training, and 15 min of balance exercises, on endurance (peak aerobic capacity (VO2 peak), muscle strength, muscle mass, and the rate of muscle protein synthesis (basal rate and anabolic response to feeding) in nine 65- to 80-year-old, moderately frail, obese older adults. Villareal et al. (2011) found that regular strength and endurance exercises increased the mixed muscle protein fractional synthesis rate (FSR) but had no effect on the feedinginduced increase in muscle protein FSR. Fat mass decreased significantly pre training 46.8 $\pm 5.3 \mathrm{~kg}$ versus post training $45.1 \pm 4.8$. $\mathrm{kg}$, respectively $(\mathrm{p}<0.05)$ Fat free mass $(\mathrm{FFM})$ and appendicular lean body mass, lower body strength as measured by a Hoist machine including knee extension, knee flexion, and leg press 1-RM) upper body strength (with seated row, bench press, and bicep curl 1-RM) increased on average 10-20\% as a result of training. Moreover, peak aerobic exercise capacity improved by $\sim 15 \%$ from the beginning until the end of the exercise training program. Villareal et al. (2011) also argued that the training-induced increase in muscle mass was mediated by an increase in the basal rate of muscle protein synthesis rather than an increase in the anabolic response to feeding. Thus, resistance training and aerobic exercise seems to be the main catalysts here as contrasted with a modified diet.

Agergaard et al. (2017) reported that acute light load resistance exercise (LL-RE) increased skeletal muscle fractional synthetic rate (FSR) which was used for evaluating 
the anabolic response and hypertrophy signaling by select targets of the mechanistic target of rapamycin complex 1 (mTORC1)-signaling cascade in untrained healthy elderly (>65-yr-old) men. LL-RE was performed at $16 \%$ of their 1 RM, with 10 sets of 36 repetitions with $30 \mathrm{sec}$ rest between each set in this study.

Kumar et al. (2009) reported that the acute bouts of resistance exercise at different intensities ( 3 sets $\times 27$ repetitions (reps) at $20 \% 1$ RM, 3 sets $\times 14$ reps at $40 \% 1 \mathrm{RM}, 3$ sets $\times$ 9 reps at $60 \% 1 \mathrm{RM}, 3$ sets $\times 8$ reps at $75 \% 1 \mathrm{RM}$ and at 6 sets $\times 3$ reps $90 \% 1 \mathrm{RM})$ stimulates myofibrillar protein synthesis and anabolic signaling in a dose-dependent manner in both young (age: $24 \pm 6$ years) and old men (age: $70 \pm 5$ years) in post-absorptive state.

We concluded that resistance training performed at different velocities affect muscle protein synthesis (MPS) both acutely and with long termed conditions in elderly.

\subsection{Bone Turnover}

Demontiero, Vidal \& Duque (2012, pp.61) reported that "as a result of the aging process, bone tissue deteriorates in composition, structure and function, which predisposes it to osteoporosis" (Demontiero, Vidal \& Duque, 2012). Osteoporosis is commonly defined as deterioration in bone mass and micro-architecture, with increasing risk to fragility fractures (Raisz and Rodan, 2003, pp.15). Exercise and Sports Science Australia (ESSA) recommended that an exercise program of moderate to high-impact weight-bearing activities, high intensity progressive resistance training and balance training forms the basis for the prevention and management of osteoporosis (Beck et al., 2017). Some authors argued that regular walking for leisure in isolation and other forms of low or non-impact aerobics activities such as cycling and swimming have been shown to have little or no effect on preventing age-related bone loss in postmenopausal women (Greenway, Walkley \& Rich, 2012; Ma, Wu \& He, 2013)

Vincent \& Braith (2002) examined the effect of 6 months of high- or low-intensity resistance exercise (REX) on bone turnover in adults aged 60-83 years old. Sixty-two men and women ( $64 \pm 6$ year) were randomly assigned to a control (CON, $n=16)$, low-intensity exercise (LEX, n=24), or high-intensity exercise (HEX, n=22) group. Subjects participated in 6 months of progressive REX training and trained at either 50\% of their 1RM (lowintensity group) for 13 repetitions (LEX) or $80 \%$ of their 1RM (high intensity group) for 8 repetitions (HEX), 3 times $w^{-1}$ for 24 weeks. Subjects performed one set each of 12 different exercises. Bone mineral density (BMD) was measured for total body, femoral neck, and lumbar spine using dual energy X-ray absorptiometry (DXA). Serum levels of bone-specific alkaline phosphatase (BAP), osteocalcin (OC), and serum pyridinoline cross-links (PYD) were also measured. Bone mineral density of the femoral neck increased significantly $(1.96 \%)$ in the HEX group. No other significant changes for BMD were found. OC increased by $25.1 \%$ and $39.0 \%$ in the LEX and HEX groups, respectively. BAP also increased significantly $(7.1 \%)$ in the HEX group. In conclusion, the data indicated that the high-intensity REX training is successful in improving BMD of the femoral neck in healthy 
elderly subjects. It also suggested that resistance exercise increases bone turnover, which over time may lead to further changes in bone mineral density (Vincent \& Braith, 2002).

In a study by Marques et al. (2013), the effects of a 32-week exercise program, which emphasized balance on lower-extremity muscle strength, bone mineral density, and serum levels of bone metabolism and inflammatory markers in older adults, were investigated. The study had forty-seven healthy older adults (women=24, men=23; mean age: 68.2 years old) participating in an exercise intervention for 3 times/week and 60 minutes per session. Participants performed resistance training (2 days/week, Mondays and Fridays) at $75-80 \%$ of their $1 \mathrm{RM}$, and a multicomponent weight-bearing impact exercise training (1 day/week, Wednesdays). Outcome measures included lumbar spine and proximal femoral bone mineral density (BMD), dynamic balance, muscle strength, serum levels of bone metabolism markers [osteocalcina (OC), C-terminal telopeptide of Type I collagen (CTX), osteoprotegerin (OPG) and receptor activator of nuclear factor kappa B ligand (RANKL)] and inflammatory markers [high sensitive (hs)-C-reactive protein (CRP), interleukin (IL)-6, tumor necrosis factor (TNF)- $\alpha$, and interferon (IFN)- $\gamma$ ]. After 32 weeks, both men and women increased dynamic balance $(6.4 \%)$, muscle strength $(11.0 \%)$ and trochanter $(0.7 \%)$, intertrochanter $(0.7 \%)$, total hip $(0.6 \%)$, and lumbar spine BMD (1.7\%), while OC, CTX, OPG and RANKL levels remained unchanged. Additionally, the hs-CRP and IFN- $\gamma$ levels decreased, TNF- $\alpha$ levels were unchanged, and a decrease in IL-6 levels was only observed in men. The researchers suggest that the combined protocol reduced inflammation and increased $\mathrm{BMD}$, balance, and lower-extremity muscle strength, despite having little effect on bone metabolism markers, thus reinforcing the role of exercise to counteract age-related inflammation, and the muscle strength, balance and BMD reduction (Marques et al., 2013).

In the study of Huovinen et al. (2016), 37 elderly women (resistance training group) (mean age $71.9 \pm 3.1$ years) with decreased muscle strength participated in a resistance training intervention three times per week with 60 min per session for 16 weeks. Resistance training group continued with different resistance exercises targeting large muscle groups of the lower and upper body (e.g., leg presses, chest presses, seated rows, abdominal crunches, back extensions, seated leg curls and hip abductions). At each station, subjects completed three sets of 8-15 repetitions with a load that corresponded to $50-80 \%$ of estimated 1RM. Resistance training group and control group tested for total hip BMD with quantitative computed tomography (QCT), bone markers (sclerostin, osteocalcin, CTX, PINP, IGF-1, 25(OH)-D) at baseline and post-intervention (16 weeks later). Authors (2016) reported that resistance training seemed to increase total hip BMD by $6 \%(p=0.005)$, sclerostin $(p<0.001)$ and total osteocalcin $(p=0.04)$ while other bone markers remained unchanged.

\subsection{Gait \& Balance}

Age-related decline in muscle power may be an early indicator of balance deficits and fall risk even in healthy, non-frail adults (Orr et al., 2006, pp.78) It has been recorded that 33\% 
Ozan Atalağ, Cem Kurt, Lincoln A. Gotshalk, Richard E. J. Shanklin, Jenna H. Aina, Ian McQuate AGING \& MUSCULAR FUNCTION: A SELECTED REVIEW OF LITERATURE WITH EMPHASIS ON CARDIORESPIRATORY ENDURANCE AND FUNCTIONAL PERFORMANCE RESPONSE TO RESISTANCE EXERCISE

of individuals 65 years and older who have fallen once, have a 50\% higher chance of falling again (Gschwind et al., 2013). Several most common factors that increase falling incidents include muscle weakness, balance deficiencies, and gait instabilities, polypharmacy, visual impairments, cognitive decline especially attention and executive dysfunction (Ambrose, Paul and Harsdorf, 2013).

Paterson et al. (2010) examined the effect of power training on balance performance in healthy older adults. One-hundred twelve community-dwelling healthy older adults (69 \pm 6 years) were randomly assigned to 8-12 weeks of power training at a low intensity $(20 \%$ of $1 \mathrm{RM})$, medium intensity $(50 \%$ of $1 \mathrm{RM})$, or high intensity $(80 \%$ of $1 \mathrm{RM})$, or a nontraining control group. Participants trained twice weekly doing five different exercises for 3 sets of 8 rapid concentric/slow eccentric repetitions using pneumatic resistance machines. Muscle performance (strength, power, endurance, contraction velocity), balance, and body composition were measured. Participants who underwent power training improved balance performance when compared to controls with the low intensity group having the greatest improvement. These results lead to conclusions that power training can be used to improve balance, particularly by using a low load, high velocity regimen in older adults with relatively low muscle power and slow muscular contraction.

Wollesen et al. (2017) compared Balance and task managing training (BDT) (motor cognitive balance training) versus strength and resistance training (ST) in walking performance (step length and gait-line) and fear of fall in older adults (mean age $=72.0 \pm$ 5.0 years). In this study, the BDT group $(n=29)$ performed task management training using balance and coordination tasks while the ST group $(n=23)$ performed resistance training only. Both ST and BDT consisted of 12 monthly sessions, 60 min each, for 12 weeks. Wollesen et al. (2017) found that both ST and BDT reduced fear of fall. Both groups also improved gait performance for step length $(\mathrm{p}<0.001)$ and gait-line $(\mathrm{ST}: \mathrm{p}<0.01$; BDT: $p<0.05)$. The BDT training group showed greater improvements in step length $(p<0.001)$ and gait-line $(\mathrm{p}<0.01)$ during dual task walking.

In another study by Henderson et al. (2017) aerobic training (AR) versus resistance exercise training (RT) were compared in terms of short physical battery score (SPPB), usual-pace gait speed and fast-pace gait speed in overweight and obese [body mass index $\left.(\mathrm{BMI}) \geq 27.0 \mathrm{~kg} / \mathrm{m}^{2}\right]$, sedentary men and women aged 65-79 years. SPPB included a standing balance test (holding a side by side, semi-tandem and tandem position for a maximum of $10 \mathrm{~s}$ ), usual-pace gait speed over a 4-m course and time to completion of five repeated chair stands without use of the arms. The AR group walked on a treadmill at an intensity of $65-70 \%$ of heart rate reserve for $15-30 \mathrm{~min}$ (from the first week to last week of the study), 4 days/week. The RT group completed 3 sets of 10 repetitions at $70 \% 1 \mathrm{RM}$ using Nautilus resistance machines comprised of leg press, leg extension, seated leg curl, seated calf, incline press, compound row, triceps press, and biceps curl exercises and exercised 3 days/week. The results of study reported that both AR and RT resulted in similar, improvements in usual pace gait speed $(0.08 \mathrm{~m} / \mathrm{s})$, short physical performance 
Ozan Atalağ, Cem Kurt, Lincoln A. Gotshalk, Richard E. J. Shanklin, Jenna H. Aina, Ian McQuate

AGING \& MUSCULAR FUNCTION: A SELECTED REVIEW OF LITERATURE

WITH EMPHASIS ON CARDIORESPIRATORY ENDURANCE AND FUNCTIONAL

PERFORMANCE RESPONSE TO RESISTANCE EXERCISE

battery score ( 0.5 points) and chair rise time ( $>1 \mathrm{~s})$. However, only AR improved fast pace gait speed $(0.11 \mathrm{~m} / \mathrm{s})$.

Paterson \& Warburton (2010) analyzed systematic reviews of the relationship between physical activity and healthy community-dwelling older adults ( $>65$ years) and the outcomes of functional limitations, disability or loss of independence. Their analysis included cohorts that had an outcome related to the functional independence or cognitive function, as well as exercise training interventions that reported a functional outcome. After reviewing 66 studies that met their criterion guidelines, the authors concluded that there is a consistency in findings across studies and a range of outcome measures related to functional independence. Paterson \& Warburton (2010) concluded that regular aerobic activity and short-term exercise programs showed a reduced risk of functional limitations and disability in older age. Specifically, with regard to the parameter of gait and balance, Paterson \& Warburton (2010) concluded that, "Exercise training interventions that supplemented the aerobic exercise by including twice weekly bouts of "resistance" training of major muscle groups support the notion that resistance exercise can be used as an adjunct to aerobic physical activity to counter the age-related loss of muscle mass, maintain the strength and power needed to complete daily activities, and prevent falls.

A recent study conducted by Pirauá et al. (2019) aimed to evaluate the effects of 24 weeks of strength training on stable (ST) and unstable surfaces (UST) on the functional mobility, balance, and concern about falling in healthy older adults who are younger than 70. Study sample consisted of 64 community-dwelling older adults (58 female and 6 male; $67.79 \pm 5.26$ years; $68.44 \pm 12.17$ years) (Pirauá et al., 2019). Participants were grouped as: stable surface strength training (ST, $n=22)$, unstable surface strength training (UST, n=22) or control (CG, $n=20)$. The ST group exercised for 24 weeks (3 times per week) on alternating days. The training program consisted of seven exercises including: $45^{\circ}$ range of motion leg press, horizontal dumbbell chest press, unilateral row with dumbbells, plank, bridge, and abdominal exercises. The prescription involved 2-5 sets and repetitions ranging from 7-12 repetitions. UST group used the same training protocol as the ST group; however, unstable devices (i.e., BOSU ${ }^{\circledR}$ ball, balance disk, and Swiss ball) were included to increase the balance component as well as neuromotor and proprioceptive demand. Participants were tested on balance, which was determined by the Brazilian version of Berg Balance Scale (BBS); functional mobility, which was assessed by the Timed Up and Go (TUG) test and Sitting and Rising Test (SRT); and concern about falling evaluated by the Brazilian version of Falls Efficacy Scale - International (FES) at baseline and at the end of 24 weeks. Results indicate that both ST and UST interventions promote beneficial effects on dynamic balance, with UST also improving functional mobility and decreasing the incidence of falling in older adults. Pirauá et al. (2019) posit that UST is an effective strategy to improve balance, functional mobility, and reduce the incidence of falling in older people.

As shown in the previous studies, it seems that resistance training (low load high velocity, high load slow velocity, strength training on stable surface, strength training on 
unstable surface) or combined aerobic and resistance training may positively affect gait, balance and functional performance. However, it remains uncertain whether resistance training alone is sufficient to prevent falls in older adults.

\subsection{Neuromuscular Adaptations}

Efficient neuromuscular adaptations in elderly populations result in improved muscle strength, power development, and cardiorespiratory function (Cadore et al., 2013) As mentioned earlier, traditionally, endurance training improved cardiovascular function while resistance training was used to increase muscle strength and power (Cheng, Chiu, \& Su, 2019; Maiorana et al., 2001; Pollock et al., 1998). Concurrent studies (Cadore et al., 2010; Holviala et al., 2010; Hurst et al., 2019; Izquierdo et al., 2004; Karavirta et al., 2011; Wood et al., 2001) found improvement when using a combination of strength training and endurance training to preserve the capacities of both areas. However, studies found that due to the "interference effect" strength gains are lower when performing a combination of both, compared to doing either strength or endurance training alone (Bell et al., 1997; Cadore et al., 2010;).

A study by Walker et al. (2013) tested 37 subjects (aged $65 \pm 4$ years old) who were randomly assigned to the constant training group (CTG), variable training group (VTG) or a non-training control group. The CTG used machines where lever arm distance is maintained throughout the range of motion. The VTG, on the other hand, used variable resistance machines where the lever arm distance is manipulated to provide greater resistance during certain phases of the range of motion (Frost, Cronin \& Newton, 2010). Both groups completed a 20-week, medium intensity, high volume resistance training program. The researchers tested the maximum bilateral concentric and isometric force production of the leg extensors as well as repetitions-to failure pre-, mid- and posttraining. Walker et al. (2013) concluded that both training groups increased force production of the leg extensors (variable: $26 \mathrm{~kg}, 95 \% \mathrm{CI}=12-39, \mathrm{p}<0.01$; constant: $31 \mathrm{~kg}$, $95 \% \mathrm{CI}=19-43, \mathrm{p}<0.01)$ and the vastus lateralis muscle's cross-sectional area significantly (variable: $1.5 \mathrm{~cm} \mathrm{2,} 95 \% \mathrm{CI}=0.03-3.1, \mathrm{p}=0.046$; constant: $3 \mathrm{~cm} 2,95 \% \mathrm{CI}=1.2-4.8, \mathrm{p}=$ 0.002).When analyzing repetitions to failure, only the VTG improved performance significantly $(704 \mathrm{~kg}, 95 \% \mathrm{CI}=45-1364, \mathrm{p}=0.035)$. Fatigue-resistance properties, such as the repetition to failure test were also improved, in the VTG which may be an important adaptation to maintain exercise and functional capacity in older individuals (Walker et al. 2013).

Hortobágyi et al. (2001) argued that low intensity (bilateral supine leg press of 40 $\% 1 \mathrm{RM}, 5$ sets, 8-12 repetitions) and high intensity (bilateral supine leg press of $80 \% 1$ RM, 5 sets, 4-6 repetitions) strength training performed for 10 weeks, 3 days a week, are equally effective in partially restoring elderly adults' (age, 72 years) maximal strength and submaximal force control.

A meta-analysis the effect of high-intensity progressive resistance strength training on strength, function, mood, quality of life of older adults published by Raymond et al. 
(2013) reported that using high-intensity progressive resistance exercises may improve lower limb strength more than using lower intensities. They also indicated that the training volume has an important effect on the strength gains achieved as well. However, other than strength, training intensity did not appear to impact greatly on outcomes of muscular power and endurance of lower extremities, cardiovascular fitness, knee flexion and hip extension range of motion, functional performance, fall risk and quality of life in older adults (mean age $\geq 65 y$ ).

Another review article of the intensity and effects of strength training done by Mayer et al. (2011) also reported that progressive strength training in the elderly is effective, even at higher intensities, to reduce sarcopenia, and to retain motor function.

Although there is clear evidence indicating the positive effects of both low and high volume resistance training, future research needs to establish the optimal volume depending on the individual or population based goals such as functional ability and neuromuscular gain in the elderly.

\section{Conclusion}

The literature presented demonstrates that human aging can be viewed as a disease, result of disuse, or a genetic condition, which, in theory, is treatable. In addition, the studies presented indicates that programs of resistance exercise have shown the ability to defer, retard, reverse, or in a manner provide an improvement in many of the declines which are associated with the aging process.

Further research into human aging is necessary, particularly with subjects of the "baby boomer" population, often defined as people born between 1947 and 1963. This population will represent the largest population group in history. Thus, with such a large population influx streaming into the "older" adult phases of their life, research pertaining to senescence will be a crucial factor in allowing the aged population to sustain a quality of life that is not only physiologically beneficial, but crucial to their cognitive and sociological wellbeing.

Furthermore, a single study cannot completely answer the effects of resistance training on subject areas of the aging population. It is understood that there are many factors that contribute to the outcome of these studies. Variables include gender, age group, ethnicity, healthy versus frail elderly subjects, volume, intensity, frequency, and duration of the training program, supervised versus unsupervised, detraining, types of exercises, and aerobic, strength training or a combination of both. These studies can only suggest the positive influences which resistance training can possibly offer the growing elderly population.

According to the studies examined in this review paper, we concluded that performing low load high velocity strength/resistance training combined with endurance training might be a better training strategy than alone endurance training or strength/resistance training in improving cardiovascular fitness, functional capacity and 
musculoskeletal heath in the elderly. Thus, elderly populations might be encouraged to participate in a concurrent training of cardiovascular endurance and muscle strength and endurance or either training modality alone to delay, or even reverse negative changes that are associated with aging

\section{Conflict of Interest Statement}

The authors declare no conflicts of interests.

\section{About the Authors}

Dr. Ozan Atalağ is an associate professor Department of Kinesiology and Exercise Sciences, University of Hawai'i at Hilo, USA. His main research interest is in the area of strength \& conditioning and enhancement of athletic performance.

Dr. Cem Kurt is an associate professor Department of Coaching Education, School of Kirkpinar Physical Education and Sports in Trakya University, Turkey. His current research interest is the relationship between warm up and superior performance. Dr. Lincoln A. Gotshalk is a full professor Department of Kinesiology and Exercise Sciences, University of Hawai'i at Hilo, USA. His current research interest is how exercise impacts cancer survivors' physical and mental health, and what motivational mechanisms can help inactive cancer survivors begin to exercise.

Richard E. J. Shanklin, Jenna H. Aina, Ian McQuate were undergraduate students at Department of Kinesiology and Exercise Sciences, University of Hawai'i at Hilo, USA.

\section{References}

Agergaard, J., Bülow, J., Jensen, J. K., Reitelseder, S., Drummond, M. J., Schjerling, P., \& Holm, L. (2017). Light-load resistance exercise increases muscle protein synthesis and hypertrophy signaling in elderly men. American Journal of PhysiologyEndocrinology and Metabolism, 312(4), E326-E338.

Allen, J. D., Robbins, J. L., VanBruggen, M. D., Credeur, D. P., Johannsen, N. M., Earnest, C. P., \& Kraus, W. E. (2013). Unlocking the barriers to improved functional capacity in the elderly: Rationale and design for the "Fit for Life trial.". Contemporary Clinical Trials, 36(1), 266-275.

Ambrose, A. F., Paul, G., \& Hausdorff, J. M. (2013). Risk factors for falls among older adults: A review of the literature. Maturitas, 75(1), 51-61.

Beck, B. R., Daly, R. M., Singh, M. A. F., \& Taaffe, D. R. (2017). Exercise and Sports Science Australia (ESSA) position statement on exercise prescription for the prevention and management of osteoporosis. Journal of Science and Medicine in Sport, 20(5), 438445 . 
Bell, G., Syrotuik, D., Socha, T., Maclean, I., \& Quinney, H. A. (1997). Effect of strength training and concurrent strength and endurance training on strength, testosterone, and cortisol. The Journal of Strength $\mathcal{E}$ and Conditioning Research, 11(1), 57-64.

Blumenthal, J. A., Smith, P. J., \& Hoffman, B. M. (2012). Is exercise a viable treatment for depression? ACSM's Health \& Fitness Journal, 16(4), 14.

Bottaro, M., Machado, S. N., Nogueira, W., Scales, R., \& Veloso, J. (2007). Effect of high versus low-velocity resistance training on muscular fitness and functional performance in older men. European Journal of Applied Physiology, 99(3), 257-264.

Bowden Davies, K. A., Pickles, S., Sprung, V. S., Kemp, G. J., Alam, U., Moore, D. R., \& Cuthbertson, D. J. (2019). Reduced physical activity in young and older adults: Metabolic and musculoskeletal implications. Therapeutic Advances in Endocrinology and Metabolism, 10. doi:10.1177/2042018819888824

Braith, R. W., \& Stewart, K. J. (2006). Resistance exercise training: Its role in the prevention of cardiovascular disease. Circulation, 113(22), 2642-2650.

Cadore, E. L., Pinto, R. S., Teodoro, J. D., Da Silva, L. X. N., Menger, E., Alberton, C. L., \& Izquierdo, M. (2018). Cardiorespiratory adaptations in elderly men following different concurrent training regimes. The Journal of Nutrition, Health $\mathcal{E}$ Aging, 22(4), 483-490.

Cadore, E. L., Pinto, R. S., Lhullier, F. L. R., Correa, C. S., Alberton, C. L., Pinto, S. S., \& Kruel, L. F. M. (2010). Physiological effects of concurrent training in elderly men. International Journal of Sports Medicine, 31(10), 689-697.

Callahan, D., Phillips, E., Carabello, R., Frontera, W. R., \& Fielding, R. A. (2007). Assessment of lower extremity muscle power in functionally-limited elders. Aging Clinical and Experimental Research, 19(3), 194-199.

Centers for Disease Control and Prevention (CDC). (2004). Strength training among adults aged $>/=65$ years--United States, 2001. MMWR. Morbidity and Mortality Weekly Report, 53(2), 25.

Cheng, J. C., Chiu, C. Y., \& Su, T. J. (2019). Training and evaluation of human cardiorespiratory endurance based on a fuzzy algorithm. International Journal of Environmental Research and Public Health, 16, 2390.

Cornelissen, V. A., Fagard, R. H., Coeckelberghs, E., \& Vanhees, L. (2011). Impact of resistance training on blood pressure and other cardiovascular risk factors: A metaanalysis of randomized, controlled trials. Hypertension, 58(5), 950-958.

Cuoco, A., Callahan, D. M., Sayers, S., Frontera, W. R., Bean, J., \& Fielding, R. A. (2004). Impact of muscle power and force on gait speed in disabled older men and women. The Journals of Gerontology Series A: Biological Sciences and Medical Sciences, 59(11), 1200-1206.

da Cunha Nascimento, D., Tibana, R. A., Benik, F. M., Fontana, K. E., Neto, F. R., de Santana, F. S., \& Balsamo, S. (2014). Sustained effect of resistance training on blood pressure and hand grip strength following a detraining period in elderly hypertensive women: A pilot study. Clinical Interventions in Aging, 9, 219. 
da Rosa Orssatto, L. B., de la Rocha Freitas, C., Shield, A. J., Pinto, R. S., \& Trajano, G. S. (2019). Effects of resistance training concentric velocity on older adults' functional capacity: A systematic review and meta-analysis of randomised trials. Experimental Gerontology, 127, 110731.

Dantas, F. F. O., Brasileiro-Santos, M. D. S., Batista, R. M. F., do Nascimento, L. S., Castellano, L. R. C., Ritti-Dias, R. M., \& Santos, A. D. C. (2016). Effect of strength training on oxidative stress and the correlation of the same with forearm vasodilatation and blood pressure of hypertensive elderly women: A randomized clinical trial. PloS one, 11(8), e0161178.

Demontiero, O., Vidal, C., \& Duque, G. (2012). Aging and bone loss: New insights for the clinician. Therapeutic Advances in Musculoskeletal Disease, 4(2), 61-76.

Distefano, G., \& Goodpaster, B. H. (2018). Effects of exercise and aging on skeletal muscle. Cold Spring Harbor Perspectives in Medicine, 8(3), a029785.

Fagard, R. H. (2011). Exercise therapy in hypertensive cardiovascular disease. Progress in Cardiovascular Diseases, 53(6), 404-411.

Fielding, R. A., LeBrasseur, N. K., Cuoco, A., Bean, J., Mizer, K., \& Singh, M. A. F. (2002). High-velocity resistance training increases skeletal muscle peak power in older women. Journal of the American Geriatrics Society, 50(4), 655-662.

Finger, D., Goltz, F. R., Umpierre, D., Meyer, E., Rosa, L. H. T., \& Schneider, C. D. (2015). Effects of protein supplementation in older adults undergoing resistance training: A systematic review and meta-analysis. Sports Medicine, 45(2), 245-255.

Fragala, M. S., Cadore, E. L., Dorgo, S., Izquierdo, M., Kraemer, W. J., Peterson, M. D., \& Ryan, E. D. (2019). Resistance training for older adults: Position statement from the national strength and conditioning association. The Journal of Strength $\mathcal{E}$ Conditioning Research, 33, (8).2019-2052.

Ferrucci, L., Baroni, M., Ranchelli, A., Lauretani, F., Maggio, M., Mecocci, P., \& Ruggiero, C. (2014). Interaction between bone and muscle in older persons with mobility limitations. Current Pharmaceutical Design, 20(19), 3178-3197.

Frontera, W. R., Meredith, C. N., O'Reilly, K. P., \& Evans, W. J. (1990). Strength training and determinants of VO2max in older men. Journal of Applied Physiology, 68(1), 329333.

Frost, D. M., Cronin, J., \& Newton, R. U. (2010). A biomechanical evaluation of resistance: fundamental concepts for training and sports performance. Sports Medicine, 40(4), 303-326.

Gray, M., Powers, M., Boyd, L., \& Garver, K. (2018). Longitudinal comparison of low-and high-velocity resistance training in relation to body composition and functional fitness of older adults. Aging Clinical and Experimental Research, 30(12), 1465-1473.

Greenway, K. G., Walkley, J. W., \& Rich, P. A. (2012). Does long-term swimming participation have a deleterious effect on the adult female skeleton? European Journal of Applied Physiology, 112(9), 3217-3225. 
Gschwind, Y. J., Kressig, R. W., Lacroix, A., Muehlbauer, T., Pfenninger, B., \& Granacher, U. (2013). A best practice fall prevention exercise program to improve balance, strength/power, and psychosocial health in older adults: Study protocol for a randomized controlled trial. BMC Geriatrics, 13(1), 105.

Guizelini, P. C., de Aguiar, R. A., Denadai, B. S., Caputo, F., \& Greco, C. C. (2018). Effect of resistance training on muscle strength and rate of force development in healthy older adults: A systematic review and meta-analysis. Experimental Gerontology, 102, 51-58.

Hamczyk, M. R., Nevado, R. M., Barettino, A., Fuster, V., \& Andrés, V. (2020). Biological versus chronological aging: JACC focus seminar. Journal of the American College of Cardiology, 75(8), 919-930.

Hasten, D. L., Pak-Loduca, J., Obert, K. A., \& Yarasheski, K. E. (2000). Resistance exercise acutely increases MHC and mixed muscle protein synthesis rates in 78-84 and 23$32 \mathrm{yr}$ olds. American Journal of Physiology-Endocrinology and Metabolism, 278(4), E620-E626.

Hazell, T., Kenno, K., \& Jakobi, J. (2007). Functional benefit of power training for older adults. Journal of Aging and Physical Activity, 15(3), 349-359.

Holviala, J., Häkkinen, A., Karavirta, L., Nyman, K., Izquierdo, M., Gorostiaga, E. M., \& Häkkinen, K. (2010). Effects of combined strength and endurance training on treadmill load carrying walking performance in aging men. The Journal of Strength $\mathcal{E}$ Conditioning Research, 24(6), 1584-1595.

Hortobagyi, T., Tunnel, D., Moody, J., Beam, S., \& DeVita, P. (2001). Low-or high-intensity strength training partially restores impaired quadriceps force accuracy and steadiness in aged adults. The Journals of Gerontology Series A: Biological Sciences and Medical Sciences, 56(1), B38-B47.

Huovinen, V., Ivaska, K. K., Kiviranta, R., Bucci, M., Lipponen, H., Sandboge, S., \& Nuutila, P. (2016). Bone mineral density is increased after a 16-week resistance training intervention in elderly women with decreased muscle strength. European Journal of Endocrinology, 175(6), 571-582.

Hurst, C., Weston, K. L., McLaren, S. J., \& Weston, M. (2019). The effects of same-session combined exercise training on cardiorespiratory and functional fitness in older adults: A systematic review and meta-analysis. Aging Clinical and Experimental Research, 31 (12), 1701-1717.

Inder, J. D., Carlson, D. J., Dieberg, G., McFarlane, J. R., Hess, N. C., \& Smart, N. A. (2016). Isometric exercise training for blood pressure management: A systematic review and meta-analysis to optimize benefit. Hypertension Research, 39(2), 88-94.

Izquierdo, M., Ibanez, J. K. H. A., Hakkinen, K., Kraemer, W. J., Larrión, J. L., \& Gorostiaga, E. M. (2004). Once weekly combined resistance and cardiovascular training in healthy older men. Medicine and Science in Sports and Exercise, 36(3), 435443. 
Jaul, E., \& Barron, J. (2017). Age-related diseases and clinical and public health implications for the 85 years old and over population. Frontiers in Public Health, 5 , 335.

Kraschnewski, J. L., Sciamanna, C. N., Poger, J. M., Rovniak, L. S., Lehman, E. B., Cooper, A. B., \& Ciccolo, J. T. (2016). Is strength training associated with mortality benefits? A 15-year cohort study of US older adults. Preventive Medicine, 87, 121-127.

Karavirta, L., Häkkinen, A., Sillanpää, E., García-López, D., Kauhanen, A., Haapasaari, A., \& Gorostiaga, E. (2011). Effects of combined endurance and strength training on muscle strength, power and hypertrophy in 40-67-year-old men. Scandinavian Journal of Medicine $\mathcal{E}$ Science in Sports, 21(3), 402-411.

Kim, H. S., \& Kim, D. G. (2013). Effect of long-term resistance exercise on body composition, blood lipid factors, and vascular compliance in the hypertensive elderly men. Journal of Exercise Rehabilitation, 9(2), 271.

Komi, P. V. (1984). Physiological and biomechanical correlates of muscle function: Effects of muscle structure and stretch - shortening cycle on force and speed. Exercise and Sport Sciences Reviews, 12(1), 81-122.

Kraemer, W. J., \& Ratamess, N. A. (2004). Fundamentals of resistance training: Progression and exercise prescription. Medicine $\mathcal{E}$ Science in Sports E Exercise, 36(4), 674-688.

Kumar, V., Selby, A., Rankin, D., Patel, R., Atherton, P., Hildebrandt, W., \& Rennie, M. J. (2009). Age-related differences in the dose-response relationship of muscle protein synthesis to resistance exercise in young and old men. The Journal of Physiology, 587(1), 211-217.

Lacroix, A., Kressig, R. W., Muehlbauer, T., Gschwind, Y. J., Pfenninger, B., Bruegger, O., \& Granacher, U. (2016). Effects of a supervised versus an unsupervised combined balance and strength training program on balance and muscle power in healthy older adults: A randomized controlled trial. Gerontology, 62(3), 275-288.

Landi, F., Calvani, R., Tosato, M., Martone, A. M., Fusco, D., Sisto, A., \& Marzetti, E. (2017). Age-related variations of muscle mass, strength, and physical performance in community-dwellers: Results from the Milan EXPO survey. Journal of the American Medical Directors Association, 18(1), 88-e17.

Lyttle, A. D., Wilson, G. J., \& Ostrowski, K. J. (1996). Enhancing performance: Maximal power versus combined weights and plyometrics training. Journal of Strength and Conditioning Research, 10, 173-179.

Ma, D., Wu, L., \& He, Z. (2013). Effects of walking on the preservation of bone mineral density in perimenopausal and postmenopausal women: A systematic review and meta-analysis. Menopause, 20(11), 1216-1226.

Maiorana, A., O'Driscoll, G., Cheetham, C., Collis, J., Goodman, C., Rankin, S., \& Green, D. (2001). Combined aerobic and resistance exercise training improves functional capacity and strength in CHF. Journal of Cardiopulmonary Rehabilitation and Prevention, 21(1), 51-52. 
Mancia, G., Fagard, R., Narkiewicz, K., Redán, J., Zanchetti, A., Böhm, M., \& Galderisi, M. (2013). Practice guidelines for the management of arterial hypertension of the European Society of Hypertension (ESH) and the European Society of Cardiology (ESC): ESH/ESC Task Force for the Management of Arterial Hypertension. Journal of Hypertension, 31(10), 1925-1938.

Martel, G. F., Hurlbut, D. E., Lott, M. E., Lemmer, J. T., Ivey, F. M., Roth, S. M., \& Hurley, B. F. (1999). Strength training normalizes resting blood pressure in 65-to 73-yearold men and women with high normal blood pressure. Journal of the American Geriatrics Society, 47(10), 1215-1221.

Marques, E. A., Mota, J., Viana, J. L., Tuna, D., Figueiredo, P., Guimarães, J. T., \& Carvalho, J. (2013). Response of bone mineral density, inflammatory cytokines, and biochemical bone markers to a 32-week combined loading exercise programme in older men and women. Archives of Gerontology and Geriatrics, 57(2), 226-233.

Mayer, F., Scharhag-Rosenberger, F., Carlsohn, A., Cassel, M., Müller, S., \& Scharhag, J. (2011). The intensity and effects of strength training in the elderly. Deutsches Ärzteblatt International, 108(21), 359.

Navaratnarajah, A., \& Jackson, S. H. (2017). The physiology of ageing. Medicine, 45(1), 610.

Olher, R. D. R. V., Bocalini, D. S., Bacurau, R. F., Rodriguez, D., Figueira Jr, A., Pontes Jr, F. L., \& Moraes, M. R. (2013). Isometric handgrip does not elicit cardiovascular overload or post-exercise hypotension in hypertensive older women. Clinical Interventions in Aging, 8, 649.

Orr, R., De Vos, N. J., Singh, N. A., Ross, D. A., Stavrinos, T. M., \& Fiatarone-Singh, M. A. (2006). Power training improves balance in healthy older adults. The Journals of Gerontology Series A: Biological Sciences and Medical Sciences, 61(1), 78-85.

Paddon-Jones, D., Campbell, W. W., Jacques, P. F., Kritchevsky, S. B., Moore, L. L., Rodriguez, N. R., \& Van Loon, L. J. (2015). Protein and healthy aging. The American Journal of Clinical Nutrition, 101(6), 1339S-1345S.

Pal, S., \& Tyler, J. K. (2016). Epigenetics and aging. Science Advances, 2(7), e1600584.

Paterson, D. H., \& Warburton, D. E. (2010). Physical activity and functional limitations in older adults: A systematic review related to Canada's Physical Activity Guidelines. International Journal of Behavioral Nutrition and Physical Activity, 7(1), 122.

Pescatello, L. S., MacDonald, H. V., Lamberti, L., \& Johnson, B. T. (2015). Exercise for hypertension: A prescription update integrating existing recommendations with emerging research. Current Hypertension Reports, 17(11), 87.

Pirauá, A. L. T., Cavalcante, B. R., de Oliveira, V. M. A., Beltrão, N. B., de Amorim Batista, G., Pitangui, A. C. R., \& de Araújo, R. C. (2019). Effect of 24-week strength training on unstable surfaces on mobility, balance, and concern about falling in older adults. Scandinavian Journal of Medicine E Science in Sports, 29(11), 1805-1812. 
Pollock, M. L., Gaesser, G. A., Butcher, J. D., Després, J. P., Dishman, R. K., Franklin, B. A., \& Garber, C. E. (1998). ACSM position stand: The recommended quantity and quality of exercise for developing and maintaining cardiorespiratory and muscular fitness, and flexibility in healthy adults. Medicine $\mathcal{E}$ Science in Sports $\mathcal{E}$ Exercise, 30(6), 975-991.

Raisz, L. G., \& Rodan, G. A. (2003). Pathogenesis of osteoporosis. Endocrinology and Metabolism Clinics of North America, 32(1), 15-24. Raymond, M. J., Bramley-Tzerefos, R. E., Jeffs, K. J., Winter, A., \& Holland, A. E. (2013). Systematic review of highintensity progressive resistance strength training of the lower limb compared with other intensities of strength training in older adults. Archives of Physical Medicine and Rehabilitation, 94(8), 1458-1472.

Rebelo-Marques, A., De Sousa Lages, A., Andrade, R., Ribeiro, C. F., Mota-Pinto, A., Carrilho, F., \& Espregueira-Mendes, J. (2018). Aging hallmarks: The benefits of physical exercise. Frontiers in Endocrinology, 9, 258.

Reid, K. F., \& Fielding, R. A. (2012). Skeletal muscle power: A critical determinant of physical functioning in older adults. Exercise and Sport Sciences Reviews, 40(1), 4.

Ren, J., \& Zhang, Y. (2018). Targeting autophagy in aging and aging-related cardiovascular diseases. Trends in Pharmacological Sciences, 39(12), 1064-1076.

Ros, M., \& Carrascosa, J. M. (2020). Current nutritional and pharmacological anti-aging interventions. Biochimica et Biophysica Acta (BBA)-Molecular Basis of Disease, 1866(3), 165612.

Stec, M. J., Thalacker-Mercer, A., Mayhew, D. L., Kelly, N. A., Tuggle, S. C., Merritt, E. K., \& Roberts, B. M. (2017). Randomized, four-arm, dose-response clinical trial to optimize resistance exercise training for older adults with age-related muscle atrophy. Experimental Gerontology, 99, 98-109.

Vincent, K. R., \& Braith, R. W. (2002). Improved Cardiorespiratory Endurance Following 6 Months of Resistance Exercise in Elderly Men and Women. Arch Intern Med, 162(6):673-678

Villareal, D. T., Smith, G. I., Sinacore, D. R., Shah, K., \& Mittendorfer, B. (2011). Regular multicomponent exercise increases physical fitness and muscle protein anabolism in frail, obese, older adults. Obesity, 19(2), 312-318.

Walker, S., Hulmi, J. J., Wernbom, M., Nyman, K., Kraemer, W. J., Ahtiainen, J. P., \& Häkkinen, K. (2013). Variable resistance training promotes greater fatigue resistance but not hypertrophy versus constant resistance training. European Journal of Applied Physiology, 113(9), 2233-2244.

Williams, B., Mancia, G., Spiering, W., Agabiti Rosei, E., Azizi, M., Burnier, M., \& Kahan, T. (2018). 2018 ESC/ESH Guidelines for the management of arterial hypertension: The Task Force for the management of arterial hypertension of the European Society of Cardiology (ESC) and the European Society of Hypertension (ESH). European Heart Journal, 39(33), 3021-3104. 
Wollesen, B., Mattes, K., Schulz, S., Bischoff, L. L., Seydell, L., Bell, J. W., \& von Duvillard, S. P. (2017). Effects of dual-task management and resistance training on gait performance in older individuals: A randomized controlled trial. Frontiers in Aging Neuroscience, 9, 415.

Wood, R. H., Reyes, R., Welsch, M. A., Favaloro-Sabatier, J., Sabatier, M., Lee, C. M., \& Hooper, P. F. (2001). Concurrent cardiovascular and resistance training in healthy older adults. Medicine and Science in Sports and Exercise, 33(10), 1751-1758.

Author(s) will retain the copyright of their published articles agreeing that a Creative Commons Attribution 4.0 International License (CC BY 4.0) terms will be applied to their work. Under the terms of this license, no permission is required from the author(s) or publisher for members of the community to copy, distribute, transmit or adapt the article content, providing a proper, prominent and unambiguous attribution to the authors in a manner that makes clear that the materials are being reused under permission of a Creative Commons License. Views, opinions and conclusions expressed in this research article are views, opinions and conclusions of the author(s). Open Access Publishing Group and European Journal of Fitness, Nutrition and Sport Medicine Studies shall not be responsible or answerable for any loss, damage or liability caused in relation to/arising out of conflicts of interest, copyright violations and inappropriate or inaccurate use of any kind content related or integrated into the research work. All the published works are meeting the Open Access Publishing requirements and can be freely accessed, shared, modified, distributed and used in educational, commercial and non-commercial purposes under a Creative Commons Attribution 4.0 International License (CC BY 4.0). 